\title{
Re-bleeding After Endoscopic Hemostasis for Peptic Ulcer Bleeding: Is eNough SAID or Are Other Factors Important?
}

\author{
Mitsuhiro Fujishiro ${ }^{1}$ Yuichi Ikeda ${ }^{2}$
}

Published online: 12 March 2016

(c) Springer Science+Business Media New York 2016

Peptic ulcer bleeding (PUB) remains a major problem worldwide, with 28-59\% (duodenal ulcer 17-37\% and gastric ulcer 11-24\%) of acute non-variceal upper gastrointestinal (GI) bleeding episodes attributable to PUB [1, $2]$. Due to the increasing numbers of elderly patients with multiple comorbidities, PUB episodes are becoming more clinically complex. Antiplatelet medication, an established treatment for secondary prevention of thromboembolic events in patients at high cardiovascular risk [3], further complicates the management of PUB due to its need to be stopped in order to lessen bleeding intensity and for preventing re-bleeding, a recommendation based on the significantly higher re-bleeding rates reported in antiplatelet drug users than in nonusers [4]. Despite its acceptance as a major bleeding risk factor, others may also be important.

In this issue of Digestive Diseases and Sciences, Kim and coworkers report that chronic kidney disease (CKD) is an important risk factor for re-bleeding after successful endoscopic hemostasis for PUB [4], a somewhat inconvenient finding given the few options available for modifying CKD. A cohort study using Taiwan's National Health Insurance research database correlated increasing risk of PUB with worsening CKD [5]. Huang et al. [5] postulated several mechanisms by which $\mathrm{CKD}$ contributes to the risk of PUB: delayed gastric emptying, higher gastrointestinal hormone release (including gastrin) which increases the

Mitsuhiro Fujishiro

mtfujish@gmail.com; mtfujish-kkr@umin.ac.jp

1 Department of Endoscopy and Endoscopic Surgery, Graduate School of Medicine, The University of Tokyo, 7-3-1, Hongo, Bunkyo-ku, Tokyo 113-8655, Japan

2 Department of Cardiovascular Medicine, The University of Tokyo, Tokyo, Japan rate of gastric acid secretion, and finally the anti-inflammatory effect of residual renal function, which when unopposed may damage the gastric or small intestinal mucosa. Although theses risks are thought to be for the initial bleeding episode rather than for re-bleeding, we speculate that the acceleration of peptic ulcer (PU) healing is more important after successful hemostasis than is the bleeding tendency caused by antiplatelet drugs. Nevertheless, risk factors generated by a cohort study as opposed to a clinical trial must be interpreted with care. Furthermore, although it is not known whether CKD is causative for PUB risk or whether it merely is correlative, a previous meta-analysis revealed that major predictors for re-bleeding in patients receiving endoscopic therapy are hemodynamic instability, active bleeding at endoscopy, large ulcer size, ulcer location, hemoglobin value and the need for transfusion [6], factors which were not analyzed as possible risk factors by Kim et al. Levels of endoscopists and hemostatic methods were included in their risk analyses, and these were not significant risk factors. Although endoscopic hemostasis is considered to be technically demanding and need expertise, technical aspects would be eliminated when hemostasis is completed with an appropriate method (either clip or thermocoagulation, with epinephrine) by an appropriate endoscopist. Kim et al. clearly showed that trainees who experienced more than 3000 esophagogastroduodenoscopies should be appropriate as an operator of hemostasis, resulting in similar efficacy and safety with experts who experienced more than 100 endoscopic hemostatic procedures.

Although much data support the observation that aspirin damages the gastrointestinal mucosa [7], thienopyridine derivatives such as clopidogrel do not damage the mucosa as far as is known. Yet, Kim et al. reported that in terms of re-bleeding, there was no difference among aspirin users, 
clopidogrel users, and dual antiplatelet users, an observation that could be explained by the healing effect of proton pump inhibitors (PPI) especially in the case of aspirin, which evens the bleeding risk between aspirin and thienopyridine, a worthy topic for future studies.

Another discussion point is the fact that second-look endoscopy (SLE) was performed in all cases. In a previous study from our group using patients with artificial ulcers that formed after gastric endoscopic submucosal dissection (ESD), second-look endoscopy was not necessary [8]. Although the situation between artificial ulcers and PUs might be different, there is another concern of cost-effectiveness even in the case of PUs, since performance of SLE was only cost-effective after therapeutic endoscopy if the risk of re-bleeding was $>31 \%$ according to one study [9]. Furthermore, our study revealed that SLE group showed a higher re-bleeding rate than non-SLE group (5.4 vs. $3.8 \%$ ) [8]. Endoscopic insertion in and of itself might induce rebleeding, leaving us to conclude that SLE even in the case of PUB should be reserved for patients in whom rebleeding is strongly suspected by clinical or by laboratory data. Further discussion on significance of SLE is necessary, especially in patients with high re-bleeding risks, including CKD.

Kim et al. further revealed that the resumption of antiplatelet drug should occur after 3 days following the index endoscopy. According to Japanese guidelines, after temporary withdrawal of antithrombotic therapy, the same regimen should be re-established as soon as hemostasis has been confirmed [10], although ongoing monitoring for signs of bleeding is required after resumption. Since immediate resumption of antithrombotic therapy after gastroenterological endoscopic procedures might increase the risk of post-procedural bleeding for $>2$ weeks after the procedure, it is important to ensure that the patient is fully informed of these risks prior to giving written consent. Although Kim et al. recommended delaying the resumption of antithrombotic therapy until 3 days after achieving hemostasis, in patients with elevated thromboembolic risk factors, earlier resumption of antithrombotic therapy in the presence of frequent clinical and laboratory monitoring for re-bleeding appears to be an acceptable alternative. The timing of resumption of antithrombotic therapy after achieving hemostasis is also worthy of further study in which re-bleeding and thromboembolic risk are considered.
In conclusion, risks of re-bleeding after successful endoscopic hemostasis are similar between aspirin, clopidogrel, and their combination in the PPI era, although the re-bleeding rate of antiplatelet drug users was significantly higher than that of nonusers. CKD is a major risk factors for re-bleeding after endoscopic hemostasis according to epidemiological studies $[4,5]$ which await confirmation by randomized clinical trials.

\section{Compliance with ethical standards}

Conflict of interest Mitsuhiro Fujishiro discloses that he is a principal investigator of the University of Tokyo in a collaborative research with the HOYA Pentax Company. Yuichi Ikeda has nothing to declare.

\section{References}

1. van Leerdam ME. Epidemiology of acute upper gastrointestinal bleeding. Best Pract Res Clin Gastroenterol. 2008;22:209-224.

2. Hearnshaw SA, Logan RF, Lowe D, et al. Acute upper gastrointestinal bleeding in the UK: patient characteristics, diagnoses and outcomes in the 2007 UK audit. Gut. 2011;60:1327-1335.

3. Antithrombotic Trialists' Collaboration. Collaborative metaanalysis of randomised trials of antiplatelet therapy for prevention of death, myocardial infarction, and stroke in high risk patients. BMJ. 2002;324:71-86.

4. Kim JW, Park HJ, Jang JY, et al. When should antiplatelet agents be resumed after successful endoscopic hemostasis in patients with peptic ulcer bleeding? Dig Dis Sci. (Epub ahead of print). doi:10.1007/s10620-016-4052-2.

5. Huang KW, Leu HB, Luo JC, et al. Different peptic ulcer bleeding risk in chronic kidney disease and end-stage renal disease patients receiving different dialysis. Dig Dis Sci. 2014; 59:807-813.

6. García-Iglesias P, Villoria A, Suarez D, et al. Meta-analysis: predictors of rebleeding after endoscopic treatment for bleeding peptic ulcer. Aliment Pharmacol Ther. 2011;34:888-900.

7. Iwamoto J, Saito Y, Honda A, Matsuzaki Y. Clinical features of gastroduodenal injury associated with long-term low-dose aspirin therapy. World J Gastroenterol. 2013;19:1673-1682.

8. Mochizuki S, Uedo N, Oda I, et al. Scheduled second-look endoscopy is not recommended after endoscopic submucosal dissection for gastric neoplasms (the SAFE trial): a multicentre prospective randomised controlled non-inferiority trial. Gut. 2015;64:397-405.

9. Laursen SB. Risk factors for rebleeding in peptic ulcer bleeding: a second look at second-look endoscopy. Dig Dis Sci. 2016;61:517-522.

10. Fujimoto K, Fujishiro M, Kato M, et al. Guidelines for gastroenterological endoscopy in patients undergoing antithrombotic treatment. Dig Endosc. 2014;26:1-14. 